This is a preprint version of the paper

\title{
High order asymptotic dynamics of a nonlinearly coupled electromechanical system
}

by Valeria Settimi ${ }^{1 *}$, Francesco Romeo ${ }^{1}$

${ }^{1}$ Department of Structural and Geotechnical Engineering, Sapienza University of Rome, Italy

*Corresponding author: valeria.settimi@uniroma1.it

Please cite this work as follows:

Settimi V., Romeo F., High order asymptotic dynamics of a nonlinearly coupled electromechanical system, Journal of Sound and Vibration, 432, 470-483, 2018, DOI: 10.1016/j.jsv.2018.06.046.

Publisher link and Copyright information:

You can download the final authenticated version of the paper and the supplementary material from: https://doi.org/10.1016/j.jsv.2018.06.046.

(C) 2018 Elsevier Ltd. All rights reserved.

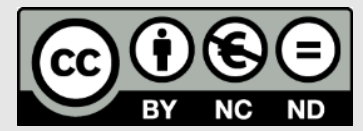

This work is licensed under the Creative Commons Attribution-NonCommercial-NoDerivatives 4.0 International License. To view a copy of this license, visit http://creativecommons.org/licenses/bync-nd/4.0/ or send a letter to Creative Commons, PO Box 1866, Mountain View, CA 94042, USA.

Licensees may copy, distribute, display and perform the work and make derivative works and remixes based on it only if they give the author or licensor the credits (attribution) in the manner specified by these.

Licensees may copy, distribute, display, and perform the work and make derivative works and remixes based on it only for non-commercial purposes.

Licensees may copy, distribute, display and perform only verbatim copies of the work, not derivative works and remixes based on it. 


\title{
High order asymptotic dynamics of a nonlinearly coupled electromechanical system
}

\author{
Valeria Settimi ${ }^{\mathrm{a}, *}$, Francesco Romeo ${ }^{\mathrm{a}}$ \\ ${ }^{a}$ Department of Structural and Geotechnical Engineering, Sapienza University of Rome, \\ 00197 Rome, Italy
}

\begin{abstract}
A nonlinearly coupled mathematical model of an electro-magneto-mechanical system is studied via the multiple scale approach in order to investigate its weakly nonlinear dynamics and analytically predict its salient features. The obtained amplitude modulation equations up to the third order perturbation allow to analytically describe the mechanical and electrical responses in terms of frequency-response curves and stability scenarios. A critical threshold of Hopf bifurcation is detected and analyzed as a function of the main system parameters. The subsequent extension of the asymptotic scheme up to the fifth order proves to grasp also the post-critical behavior, providing with the accurate identification of the amplitude of the quasiperiodic responses characterizing the unstable region of the ODEs system.
\end{abstract}

Keywords: Electromechanical system, Multiple Scale Method, Stability Analysis, Post-critical Behavior

\section{Introduction}

The nonlinear dynamics of an electromechanical system is considered in this study. The system consists of a linear oscillator nonlinearly coupled through an electromagnet to a linear electric circuit. As it is known, electromechani5 cal systems are characterized by the interaction among inertial, electric, and magnetic circuits, and they are nowadays widely used for devices that monitor and control machine and structural systems. Depending on the specific application, electromechanical devices range from macro to micro and nano scales. Irrespective of the scale, they can be modeled by lumped physical elements, 10 such as mass or inductance, for which basic dynamic principles apply, leading to coupled ordinary differential equations (ODEs) 11. The conversion between electrical and mechanical energy may take place in electromechanical motion devices through energy transfer due to coupling electromagnetic field.

\footnotetext{
${ }^{*}$ Corresponding author

Email addresses: valeria.settimi@uniroma1.it (Valeria Settimi), francesco.romeo@uniroma1.it (Francesco Romeo)
} 
In electromagnetic circuits, the dynamical equations take the form of conservation of charge and the Faraday-Henry law of flux change. If the system has a mechanical state variable, such as displacement, then the inductance may be a function of it. In such a case the magnetomotive force, induced by a current exciting a coil, acting on a ferromagnetic movable member depends on its displacement thereby triggering oscillations. By exploiting this principle, a number of devices have been proposed so far.

Magnetic solenoid actuators are electromagnetic devices that convert electrical energy into a mechanical push/pull force or motion finding a number of applications across different industries. Solenoids are variable reluctance translational machines integrating movable (plunger) and stationary (fixed) mem25 bers made from high permeability ferromagnetic materials; in these actuators the inductance and the reluctance force generated both vary nonlinearly with position [2 4. Synchronous reluctance motors are rotational electromechanical devices in which the magnetizing inductance is a function of the rotor angular displacement [3]. From a modeling perspective, in electrodynamic loudspeakers

30 it has been recently proved that the nonlinearity of inductance as function of displacement is a significant source of distortion; a linear approximation of this voice-coil inductance dependence was considered in 5 .

The nonlinear dynamics stemming from the electromagnetic coupling characterized by displacement dependent induction was also addressed by a number

35 of studies related to classical problems, such as the Bethenod's pendulum. In this system a pendulum with a ferromagnetic bob experiences growing and sustained oscillations when it is made to vary the inductance of a coil excited by high-frequency current [6]. In this work, a quadratic law for the inductance dependence on the pendulum oscillation angle was used to approximate the 40 experimental bell shaped profile. A similar electromechanical system was considered in [7. The electromechanical system consists of a linear electrical circuit whose inductance varies linearly with the position of a bar. The bar, which is made of magnetic material forming part of the inductance core, represents the mass of a damped, linear, mechanical oscillator and is forced by the pondero45 motive action of the electromagnetic field of the inductance. The nonlinear amplitude frequency response, merely of the electrical circuit, and a region of instability were analytically obtained using a modified Ritz averaging technique. Later, the same two degrees of freedom system was considered in 8, 9 as an example of self-oscillatory system. Combination frequencies arise due to the nonlinear interaction between the dynamic variables, so that the oscillations become quasiperiodic. As a result of the interaction of these oscillations with the harmonic source, energy transfer from the high-frequency (electric) source to the low-frequency (mechanical) oscillations occurs. An asymptotic approach assuming a quasiperiodic electrical response lead to obtain the slowly varying 55 functions describing amplitude and phase of the mechanical oscillator.

From the analytical point of view it is worth mentioning the similarity between the nonlinearly coupled behavior in liquid-shell systems and the electromechanical system at hand. The analogy in the governing differential equations is obtained by adopting a simplified liquid-shell system in which only one 
mode is considered 7,10 .

In 11, 12] the same electromechanical system here tackled is numerically thoroughly investigated. The occurrence of significant bifurcations marking the transition from regular to non regular responses are detected. Moreover, a wide unstable region with high-amplitude quasiperiodic and chaotic responses char-

65 acterizes the system's nonlinear dynamics. Aiming at predicting and describing this peculiar behavior while completing the outcomes presented in a companion paper, a purely analytical asymptotic approach is here proposed. This analysis turns out to be effective due to the persistence of the unstable region also for rather low values of the nonlinear coupling. In developing the asymptotic performed though a slow-fast time decomposition analysis needed to reach high orders to catch the dynamical phenomena. In the light of this, a high order Multiple Scale Method [13 15] is applied to the model. The paper is organized as follows. After the introductory section, and a brief description of the physical model under analysis in Section 2, the multiple scale formulation is presented in Sections 3 and 4 it is shown that the asymptotic approach up to the third order enables to identify the frequency response curve around the primary resonance and to analyze its stability. By extending the asymptotic approach up to the fifth order, the post-critical regime is then addressed in Section 5 , the concluding remarks are eventually reported.

\section{Modeling}

The physical model herein considered is composed of a linear oscillator nonlinearly coupled to a linear electric circuit by means of an electromagnet [1]. Under the hypothesis of linear dependence of the inductance $L$ on the mechanical displacement $x$, i.e. $L(x)=L_{0}+L_{1} x$, the equations of motion for the electro-mechanical system are:

$$
\begin{aligned}
& \ddot{x}(t)+2 \zeta_{m} \omega_{m} \dot{x}(t)+\omega_{m}^{2} x(t)=\frac{\xi \alpha \omega_{m}^{4} \dot{q}(t)^{2}}{\omega_{e}^{4}} \\
& (1+\alpha x(t)) \ddot{q}(t)+\left(2 \zeta_{e} \omega_{e}+\alpha \dot{x}(t)\right) \dot{q}(t)+\omega_{e}^{2} q(t)=\beta \omega_{e}^{2} \sin (\Omega t)
\end{aligned}
$$

where $x(t)$ and $q(t)$ are the mechanical and electrical (charge) displacements, respectively, and

$$
\omega_{m}^{2}=\frac{k}{m}, \omega_{e}^{2}=\frac{1}{L_{0} C}, \zeta_{m}=\frac{c}{2 \sqrt{k m}}, \zeta_{e}=\frac{R \sqrt{C}}{2 \sqrt{L_{0}}}, \xi=\frac{L_{0}}{2 m}, \alpha=\frac{L_{1}}{L_{0}}
$$

$m, c, k$ are the mass, viscous dissipation and stiffness parameters of the mechanical oscillator, $C, R$ are capacitance and resistance of the electrical circuit, and $\beta$ and $\Omega$ are amplitude and frequency of the harmonic voltage, respectively.

The coupling terms in (1), which represent also the sole source of nonlinearity into the model, are governed by the $\alpha$ parameter, describing the inductance coupling, therefore it is of particular interest to study the effect of such parameter on the dynamical response. Figure 11(a) displays three frequency-response 


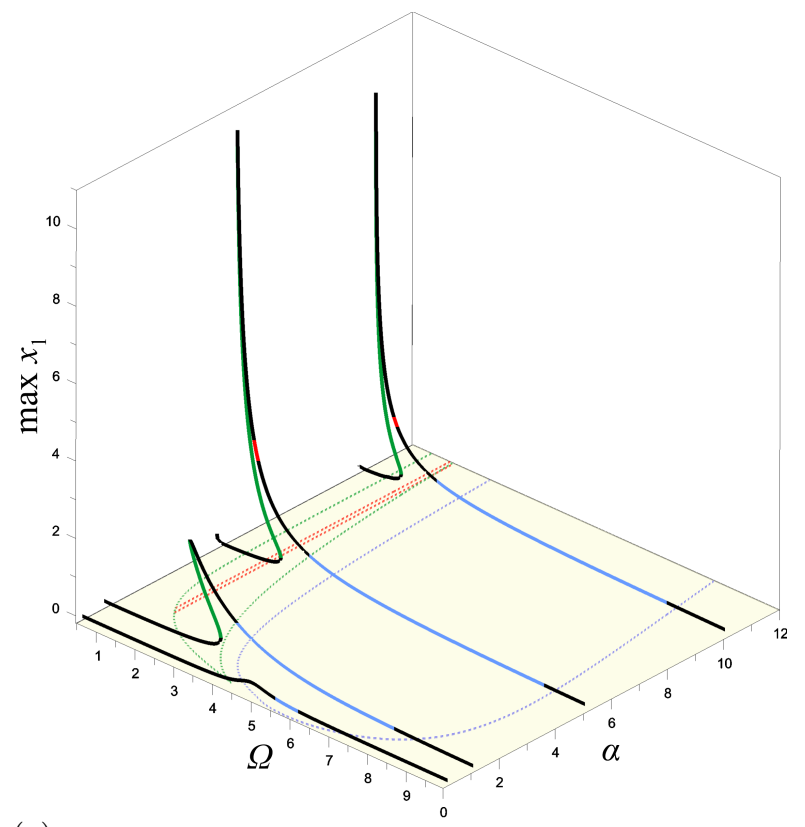

(a)

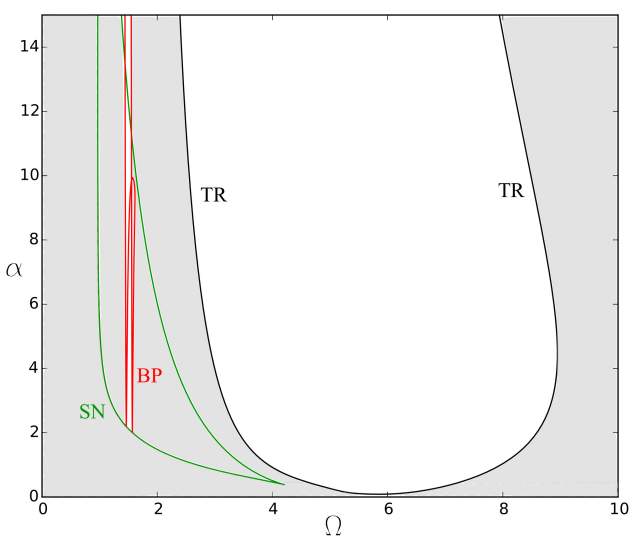

(b)

Figure 1: 3D representation of the frequency-response curves at different values of the nonlinear coupling $\alpha$, at $\mu=0.2, \beta=1.0$ (a) and relevant stability chart in the $\Omega$ - $\alpha$ plane (b). In (a), Black lines: stable solutions; Blue lines: unstable solutions through torus bifurcations; Green lines: unstable solutions through saddle-node bifurcations; Red lines: unstable solutions trough branch point bifurcations. In (b), Gray area: stable region of the period-1 solutions; black line: torus bifurcation; green line: saddle-node bifurcation; red line: branch point bifurcation.

curves obtained for different values of $\alpha$ and with the following set of parameter values: $\zeta_{m}=0.01, \zeta_{e}=0.1, \xi=5.0, \beta=1.0, \mu=\omega_{e} / \omega_{m}, \omega_{m}=1, \omega_{e}=5$. The black sections of the curves refer to the stable regions, while the gray, red and 
green ones correspond to unstable ranges delimited by tori, branch points and saddle-node bifurcations, respectively. The relevant stability chart in the $\Omega-\alpha$ plane is shown in Fig. 1(b). It can be observed that the area delimited by the torus bifurcations occurring at the right of the resonance represents the widest unstable region for the system behaving in strongly nonlinear regime (i.e. for high values of $\alpha$, see also [11]), and it turns out to be also the most robust one, persisting also for low values of the $\alpha$ parameter, down to $\alpha \cong 0.08$ where it disappears. Moreover, the occurrence of torus bifurcations points out the presence 100 of confined quasiperiodic or chaotic solutions, whose numerical characterization is presented in a companion paper. This suggests the opportunity to develop an analytical investigation, in order to get a more in-depth description of this area in terms of its existence and stability conditions, with also the possibility to identify the relevant quasiperiodic or chaotic motions.

\section{Multiple scales formulation}

The multiple scales method [16] is employed by introducing three independent time scales, i.e. the fast one, $T_{0}$, and the slower ones, $T_{1}, T_{2}$, as follows:

$$
T_{0}=t, \quad T_{1}=\epsilon t, \quad T_{2}=\epsilon^{2} t
$$

where $\epsilon$ is a small dimensionless ordering parameter and, consistently, expressing the time derivatives as

$$
\begin{aligned}
& \mathrm{d} / \mathrm{d} t=D_{0}+\epsilon D_{1}+\epsilon^{2} D_{2} \\
& \mathrm{~d}^{2} / \mathrm{d} t^{2}=D_{0}^{2}+2 \epsilon D_{0} D_{1}+\epsilon^{2} D_{1}^{2} \\
& \quad+2 \epsilon^{2} D_{0} D_{2}
\end{aligned}
$$

Both mechanical $x$ and electrical (charge) $q$ displacements are scaled as small perturbations of the reference position:

$$
\begin{aligned}
x(t)= & \epsilon x_{1}\left(T_{0}, T_{1}, T_{2}\right)+\epsilon^{2} x_{2}\left(T_{0}, T_{1}, T_{2}\right) \\
& +\epsilon^{3} x_{3}\left(T_{0}, T_{1}, T_{2}\right) \\
q(t)= & \epsilon q_{1}\left(T_{0}, T_{1}, T_{2}\right)+\epsilon^{2} q_{2}\left(T_{0}, T_{1}, T_{2}\right) \\
& +\epsilon^{3} q_{3}\left(T_{0}, T_{1}, T_{2}\right)
\end{aligned}
$$

In order to properly describe the dynamics of the weakly coupled system in presence of possible strong forcing amplitude, the parameters are scaled to obtain the forced damped electrical equation at the generating order: $\beta \rightarrow \varepsilon \hat{\beta}, \zeta_{m} \rightarrow \varepsilon^{2}$ $\hat{\zeta_{m}}$. Moreover, attention is focused on studying the primary resonance $\Omega=\omega_{e}$, with electrical-to-mechanical frequency ratio $\mu=0.2$, implying $\omega_{e}=5 \omega_{m}$. The following set of perturbation equations is obtained:

$$
\begin{aligned}
& \text { order } \epsilon^{1}: \\
& D_{0}^{2} x_{1}+\omega_{m}^{2} x_{1}=0 \\
& D_{0}^{2} q_{1}+2 \zeta_{e} \omega_{e} D_{0} q_{1}+\omega_{e}^{2} q_{1}=\omega_{e}^{2} \beta \sin \Omega t
\end{aligned}
$$




$$
\begin{aligned}
& \text { order } \epsilon^{2}: \\
& D_{0}^{2} x_{2}+\omega_{m}^{2} x_{2}=-2 D_{0} D_{1} x_{1}+\frac{\xi \alpha \omega_{m}^{4} D_{0} q_{1}^{2}}{\omega_{e}^{4}} \\
& D_{0}^{2} q_{2}+2 \zeta_{e} \omega_{e} D_{0} q_{2}+\omega_{e}^{2} q_{2}=-2 D_{0} D_{1} q_{1}-2 \zeta_{e} \omega_{e} D_{1} q_{1} \\
& -\alpha\left(D_{0}^{2} q_{1} x_{1}+D_{0} x_{1} D_{0} q_{1}\right)
\end{aligned}
$$

order $\epsilon^{3}$ :

$$
\begin{aligned}
& D_{0}^{2} x_{3}+\omega_{m}^{2} x_{3}=-2 D_{0} D_{1} x_{2}-D_{1}^{2} x_{1}-2 \zeta_{m} \omega_{m} D_{0} x_{1}-2 D_{0} D_{2} x_{1} \\
& +\frac{2 \xi \alpha \omega_{m}^{4} D_{0} q_{1} D_{0} q_{2}}{\omega_{e}^{4}}+\frac{2 \xi \alpha \omega_{m}^{4} D_{0} q_{1} D_{1} q_{1}}{\omega_{e}^{4}} \\
& D_{0}^{2} q_{3}+2 \zeta_{e} \omega_{e} D_{0} q_{3}+\omega_{e}^{2} q_{3}=-D_{1}^{2} q_{1}-2 D_{0} D_{1} q_{2}-2 D_{0} D_{2} q_{1} \\
& -\alpha\left(D_{0} x_{2} D_{0} q_{1}+D_{0} x_{1} D_{0} q_{2}+D_{0} q_{1} D_{1} x_{1}+D_{0} x_{1} D_{1} q_{1}+D_{0}^{2} q_{2} x_{1}\right. \\
& \left.+2 D_{0} D_{1} q_{1} x_{1}+D_{0}^{2} q_{1} x_{2}\right)-2 \zeta_{e} \omega_{e}\left(D_{1} q_{2}+D_{2} q_{1}\right)
\end{aligned}
$$

At the first order, the damped forced electrical equation furnishes the particular solution

$$
q_{1}=\left(\Gamma_{1}+\mathrm{i} \Gamma_{2}\right) e^{\mathrm{i} \Omega T_{0}}+\text { c.c. }
$$

where

$$
\begin{aligned}
\Gamma_{1} & =-\frac{\beta \zeta_{e} \omega_{e}^{3} \Omega}{\Omega^{4}+2\left(2 \zeta_{e}^{2}-1\right) \Omega^{2} \omega_{e}^{2}+\omega_{e}^{4}} \\
\Gamma_{2} & =\frac{\beta \omega_{e}^{2}\left(\Omega^{2}-\omega_{e}^{2}\right)}{2\left(\Omega^{4}+2\left(2 \zeta_{e}^{2}-1\right) \Omega^{2} \omega_{e}^{2}+\omega_{e}^{4}\right)}
\end{aligned}
$$

define the known amplitude, not dependent on the time scales. Differently, the solution of the mechanical equation reads

$$
x_{1}=A\left(T_{1}, T_{2}\right) e^{\mathrm{i} \omega_{m} T_{0}}+\text { c.c. }
$$

with $A\left(T_{1}, T_{2}\right)$ undetermined function of the slow time scales and c.c. complex conjugate terms (the overbar will denote the complex conjugate and $\mathrm{i}$ is the imaginary unit). By substituting $x_{1}$ and $q_{1}$ in the first equation of (6), and imposing the solvability conditions implies

$$
D_{1} A=0
$$

so that $A=A\left(T_{2}\right)$. The particular solutions at this order are

$$
\begin{aligned}
& x_{2}=\left(c_{11}+\mathrm{i} c_{12}\right) e^{2 \mathrm{i} \Omega T_{0}}+c_{13}+\text { c.c. } \\
& q_{2}=A\left(\left(c_{14}+\mathrm{i} c_{15}\right) e^{\mathrm{i}\left(\omega_{m}+\Omega\right) T_{0}}+\left(c_{16}-\mathrm{i} c_{17}\right) e^{\mathrm{i}\left(\omega_{m}-\Omega\right) T_{0}}\right)+\text { c.c. }
\end{aligned}
$$


In view of Eqs. $(8),(10),(11),(12)$, the solvability condition of the mechanical problem at the third order (7) yields

$$
\begin{aligned}
& D_{2} A=\frac{1}{\omega_{e}^{4}} \omega_{m} A\left(\xi \alpha \omega _ { m } ^ { 2 } \Omega \left(-\mathrm{i} \Omega\left(\Gamma_{1}\left(c_{14}+c_{16}+\mathrm{i}\left(c_{15}-c_{17}\right)\right)\right.\right.\right. \\
& \left.+\Gamma_{2}\left(c_{15}+c_{17}+\mathrm{i}\left(c_{16}-c_{14}\right)\right)\right)+\omega_{m}\left(-\Gamma_{2}\left(c_{14}+c_{16}+\mathrm{i}\left(c_{15}-c_{17}\right)\right)\right. \\
& \left.\left.\left.+\Gamma_{1}\left(c_{15}+c_{17}+\mathrm{i}\left(c_{16}-c_{14}\right)\right)\right)\right)-\zeta_{m} \omega_{e}^{4}\right)
\end{aligned}
$$

while the particular solutions read

$$
\begin{aligned}
& x_{3}=A\left(c_{31}+\mathrm{i} c_{32}\right) e^{\mathrm{i}\left(\omega_{m}+2 \Omega\right) T_{0}}+A\left(c_{33}+\mathrm{i} c_{34}\right) e^{\mathrm{i}\left(\omega_{m}-2 \Omega\right) T_{0}}+\text { c.c. } \\
& q_{3}=\left(c_{21}-\mathrm{i} c_{22}\right) e^{\mathrm{i} \Omega T_{0}}+\left(c_{23}+\mathrm{i} c_{24}\right) e^{3 \mathrm{i} \Omega T_{0}}+A^{2}\left(e^{\mathrm{i}\left(2 \omega_{m}+\Omega\right) T_{0}}\left(c_{27}-\mathrm{i} c_{28}\right)\right. \\
& \left.+e^{\mathrm{i}\left(2 \omega_{m}-\Omega\right) T_{0}}\left(c_{25}-\mathrm{i} c_{26}\right)\right)+A \bar{A}\left(c_{29}-\mathrm{i} c_{30}\right) e^{\mathrm{i} \Omega T_{0}}+\text { c.c. }
\end{aligned}
$$

The expressions of all $c_{i j}$ coefficients are reported in Appendix A. According to the usual reconstitution procedure, the amplitude derivatives with respect to time $t$ are obtained from (3)

$$
\dot{A}=\epsilon D_{1} A+\epsilon^{2} D_{2} A
$$

The $\epsilon$ parameter is completely reabsorbed through a backward rescaling, and, by recalling (11), the complex amplitude modulation equation (AME, or bifurcation equation) for the mechanical variable coincides with $(13)$, i.e. $\dot{A}=D_{2} A$. To conveniently express equation 13 in Cartesian form, the following coordinates transformation is applied

$$
A(t)=\frac{1}{2}(r(t)+\mathrm{i} s(t)), \quad \bar{A}(t)=\frac{1}{2}(r(t)-\mathrm{i} s(t))
$$

and, by substituting (16) in 13 the following system is obtained:

$$
\begin{aligned}
& \dot{r}=\xi \alpha^{2} \beta^{2} f_{1}(\Omega) s+\left(\xi \alpha^{2} \beta^{2} f_{2}(\Omega)-\zeta_{m} \omega_{m}\right) r \\
& \dot{s}=-\xi \alpha^{2} \beta^{2} f_{1}(\Omega) r+\left(\xi \alpha^{2} \beta^{2} f_{2}(\Omega)-\zeta_{m} \omega_{m}\right) s
\end{aligned}
$$

in which the functions $f_{1}(\Omega), f_{2}(\Omega)$ introduced are reported in Appendix A The equilibria of the asymptotic system $(17)$ coincide with the trivial rest position $(r, s)=(0,0)$, i.e. the mechanical amplitude is identically null, meaning that to obtain the frequency-response curve the amplitudes of the reconstructed electrical and mechanical solutions have to be computed. By recalling the series expansion (4) of $x$ and $q$, contributions up to the third order are taken into account by making use of (8), (10), (12), (14), and applying a backward rescaling. Guided by expressions of generating solutions (8), contributions of frequency $\omega_{m}$ and $\Omega$ are selected for the mechanical and electrical solutions, respectively, 


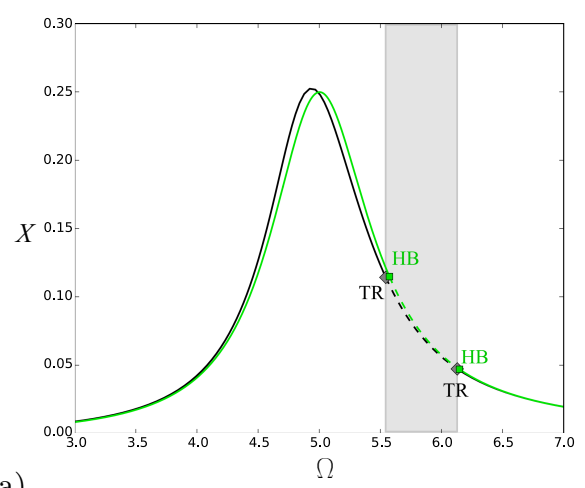

(a)

Figure 2: Comparison between numerical (black) and asymptotic (green) responses with respect to the mechanical amplitude $X$ (a) and the electrical amplitude $Q$ (b), with detection of instability regions, for $\mu=0.2$ and $\beta=1.0$. Gray diamond: torus bifurcation; green square: Hopf bifurcation.

to obtain

$$
\begin{aligned}
& x(t)=c_{13}+r \cos \left(\omega_{m} t\right)-s \sin \left(\omega_{m} t\right) \\
& q(t)=\frac{1}{2}\left(4\left(c_{21}+\Gamma_{1}\right)+c_{29}\left(r^{2}+s^{2}\right)\right) \cos (\Omega t) \\
& +\frac{1}{2}\left(4\left(c_{22}-\Gamma_{2}\right)+c_{30}\left(r^{2}+s^{2}\right)\right) \sin (\Omega t)
\end{aligned}
$$

The relevant amplitudes can be expressed as

$$
\begin{aligned}
& X=c_{13}+\sqrt{r^{2}+s^{2}} \\
& Q=\frac{1}{2} \sqrt{\left(4\left(\Gamma_{1}+c_{21}\right)+c_{29}\left(r^{2}+s^{2}\right)\right)^{2}+\left(4\left(c_{22}-\Gamma_{2}\right)+c_{30}\left(r^{2}+s^{2}\right)\right)^{2}}
\end{aligned}
$$

It is worth noting that due to the trivial equilibrium of the asymptotic system, terms related to $r$ and $s$ can be neglected.

The comparison between the analytical frequency-response curve given by Eqs. (19) and the one obtained by integrating the ODE system (1) is shown in

${ }_{110}$ Fig. 2 2 It shows a very good agreement of the results, from both a qualitative and quantitative viewpoint, highlighting the ability of the asymptotic approach to accurately identify the unstable region. To better understand its nature, the stability analysis of the asymptotic system is addressed in the next Section.

\section{Stability analysis and its dependence from system parameters}

To investigate the stability of the asymptotic responses, the eigenvalues of the Jacobian matrix of system (17) are studied:

$$
\lambda_{1,2}=\xi \alpha^{2} \beta^{2} f_{2}(\Omega)-\zeta_{m} \omega_{m} \pm \mathrm{i} \xi \alpha^{2} \beta^{2} f_{1}(\Omega)
$$




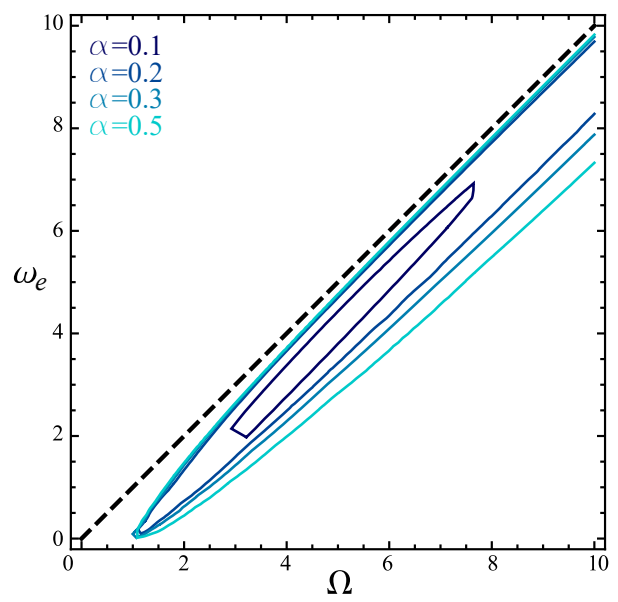

Figure 3: Analytical stability thresholds in the $\Omega-\omega_{e}$ plane, for different values of the nonlinear coupling $\alpha$.

where $f_{i}(\Omega)(i=1,2)$ are described in Appendix A. The system loses stability through a Hopf bifurcation when the real part of the complex eigenvalues becomes positive, with the critical case defined by the following equation:

$$
\xi \alpha^{2} \beta^{2} f_{2}(\Omega)-\zeta_{m} \omega_{m}=0
$$

For positive left-hand side of equation (21), the asymptotic system displays the birth of periodic solutions which, in the ODEs system, correspond to quasiperiodic and eventually chaotic responses, depending on the possible presence of other secondary bifurcations in the post-critical behavior. This post-critical behavior was detected also in 9 , where it is referred to as self-excited oscillations.

In order to get information about the position of the unstable region in the frequency-response curve, it is of interest to investigate the critical threshold (21) as a function of the electrical and external forcing frequencies. The outcomes are reported in Fig. 3 for different values of the nonlinear coupling $\alpha$; they point out that this kind of instability can occur only in the half-plane on the right of the dashed curve $\Omega=\omega_{e}$, corresponding to the right hand slope of the resonance curve (i.e. $\Omega>\omega_{e}$ ). This circumstance is also confirmed by the analyses performed in the strongly nonlinear regime (see, e.g., Fig. 1(a)).

Equation 211 can be also used to obtain indications about the values to be considered for the system parameters in order to guarantee, or alternatively ${ }_{130}$ avoid, the presence of the instability region under analysis. To focus on the role played by the electrical and mechanical damping, the behavior chart reported in Fig. 4 can be computed by zeroing the derivative of Eq. (21) with respect to $\Omega$. Thus, the chart provides the minimum values of the nonlinear coupling $\alpha$ needed to ensure the arise of the unstable area. The results highlight the major role played by the electrical damping in shifting the occurrence of instability towards higher values of the nonlinear coupling. By doubling its reference value 
(i.e. $\zeta_{e}=0.1$ ) a shift of $\alpha$ by about $124 \%$ (from 0.085 to 0.19 ) is observed; the analogous increase of the mechanical damping (i.e. from $\zeta_{m}=0.01$ to $\zeta_{m}=0.02$ ) moves the onset of instability to a $41 \%$ higher coupling value $\alpha$.

In order to validate the analytical findings, the stability threshold obtained by solving eq. 21) is compared with the torus bifurcation threshold derived from the ODEs system (1), as reported in Fig. 5 . The good agreement between analytical (dashed green) and numerical (solid gray) results is confirmed, especially for low values of the nonlinear coupling. As the $\alpha$ parameter increases, 145 slight differences can be detected, mostly located at the left side branch of the curve. They are related to the presence of the nonlinear inertial coupling, which strongly modifies the full system resonance curve peak location, which is shifted

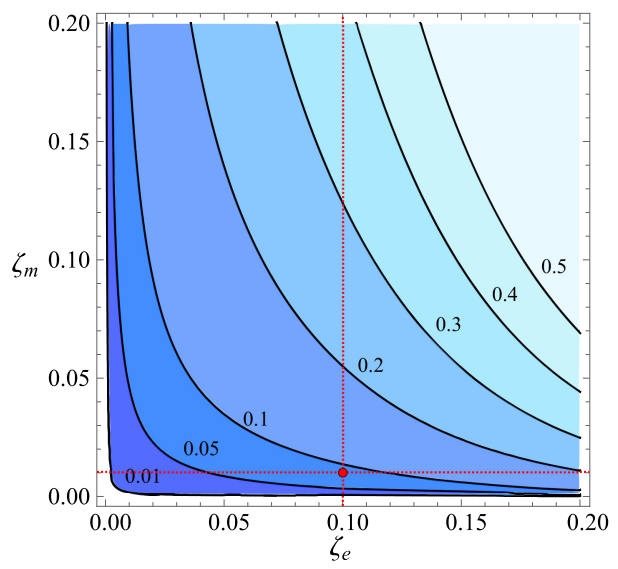

Figure 4: In the $\zeta_{e}-\zeta_{m}$ plane, analytical detection of the minimum $\alpha$ values needed to ensure the presence of the instability region.

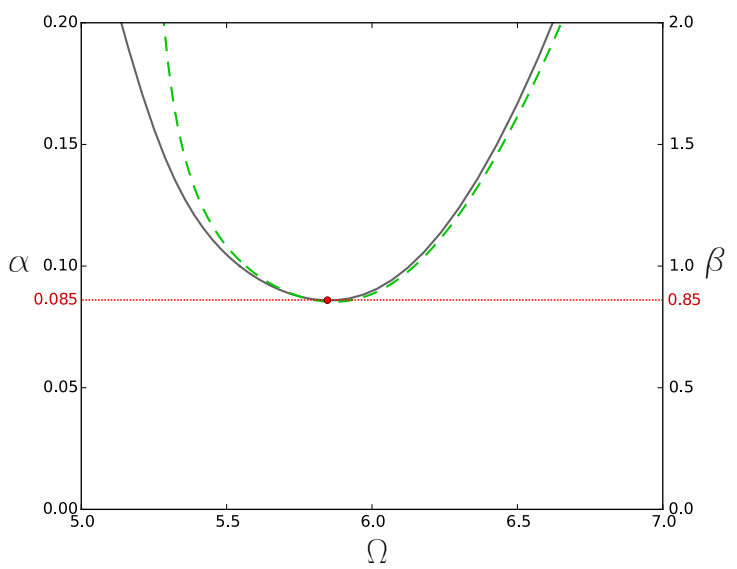

Figure 5: Asymptotic analytical (dashed green) and numerical (solid gray) stability threshold in the $\Omega-\alpha(\beta)$ plane, at $\frac{\omega_{m}}{\omega_{e}}=\mu=0.2$. 
towards lower frequency values as the coupling increases, as already illustrated in Fig. 1(a). As a consequence, the left boundary of the instability region moves accordingly. Conversely, in the formulation of the asymptotic model the nonlinearity is treated as perturbation of the underlying linear system, as such it is unable to chase the position of the resonance curve, which maintains is peak around the electrical frequency value (which coincides to 5 in the reported figures), corresponding to the left limit for the unstable area.

\section{Post-critical behavior: quasiperiodic oscillations}

In order to study the post-critical behavior of the asymptotic system arisen from the Hopf bifurcations previously detected, the multiple scales analysis is developed up to to the fifth order. Accordingly, two further slow scales are considered, i.e., $T_{3}=\epsilon^{3} t, T_{4}=\epsilon^{4} t$, and the mechanical and electrical expansions (4) are enriched by two additional orders:

$$
\begin{aligned}
x(t)= & \epsilon x_{1}\left(T_{0}, \ldots, T_{4}\right)+\epsilon^{2} x_{2}\left(T_{0}, \ldots, T_{4}\right)+\epsilon^{3} x_{3}\left(T_{0}, \ldots, T_{4}\right) \\
& +\epsilon^{4} x_{4}\left(T_{0}, \ldots, T_{4}\right)+\epsilon^{5} x_{5}\left(T_{0}, \ldots, T_{4}\right) \\
q(t)= & \epsilon q_{1}\left(T_{0}, \ldots, T_{4}\right)+\epsilon^{2} q_{2}\left(T_{0}, \ldots, T_{4}\right)+\epsilon^{3} q_{3}\left(T_{0}, \ldots, T_{4}\right) \\
& +\epsilon^{4} q_{4}\left(T_{0}, \ldots, T_{4}\right)+\epsilon^{5} q_{5}\left(T_{0}, \ldots, T_{4}\right)
\end{aligned}
$$

The perturbation equations at the fourth and fifth orders are then obtained as:

$$
\begin{aligned}
& \text { order } \epsilon^{4}: \\
& D_{0}^{2} x_{4}+x_{4} \omega_{m}^{2}=f_{4}\left(x_{1}, x_{2}, x_{3}, q_{1}, q_{2}, q_{3}\right) \\
& D_{0}^{2} q_{4}+2 \zeta_{e} \omega_{e} D_{0} q_{4}+\omega_{e}^{2} q_{4}=g_{4}\left(x_{1}, x_{2}, x_{3}, q_{1}, q_{2}, q_{3}\right)
\end{aligned}
$$

order $\epsilon^{5}$ :

$$
\begin{aligned}
& D_{0}^{2} x_{5}+\omega_{m}^{2} x_{5}=f_{5}\left(x_{1}, x_{2}, x_{3}, x_{4}, q_{1}, q_{2}, q_{3}, q_{4}\right) \\
& D_{0}^{2} q_{5}+2 \zeta_{e} \omega_{e} D_{0} q_{5}+\omega_{e}^{2} q_{5}=g_{5}\left(x_{1}, x_{2}, x_{3}, x_{4}, q_{1}, q_{2}, q_{3}, q_{4}\right)
\end{aligned}
$$

where $f_{i}, g_{i}(i=4,5)$ are given in Appendix B. Substitution into system (23) of the obtained expressions of the mechanical and electrical solutions at the 3 previous orders (8), 10], 12, (14), allows to select the secular terms at the fourth order

$$
\begin{aligned}
& \frac{2}{\omega_{e}^{4}}\left(\omega_{e}^{4}\left(D_{1} D_{2} A+\mathrm{i} \omega_{m} D_{3} A\right)+D_{1} A\left(\xi \alpha \left(-c_{15}\left(\Gamma_{1}-\mathrm{i} \Gamma_{2}\right)\right.\right.\right. \\
& \left.\left.\left.+\left(c_{16}-\mathrm{i} c_{17}\right)\left(\Gamma_{2}-\mathrm{i} \Gamma_{1}\right)+c_{14}\left(\Gamma_{2}+\mathrm{i} \Gamma_{1}\right)\right) \Omega \omega_{m}^{4}+\zeta_{m} \omega_{m} \omega_{e}^{4}\right)\right)=0
\end{aligned}
$$

which furnish, by means of 11 ,

$$
D_{3} A=0
$$


The particular solutions at this order read:

$$
\begin{aligned}
& x_{4}=\left(c_{41}-\mathrm{i} c_{42}+c_{43} A \bar{A}-\mathrm{i} c_{44} A \bar{A}\right) e^{2 \mathrm{i} \Omega T_{0}}+\left(c_{45}-\mathrm{i} c_{46}\right) e^{4 \mathrm{i} \Omega T_{0}} \\
& +\left(c_{47}-\mathrm{i} c_{48}\right) A^{2} e^{2 \mathrm{i} T_{0}\left(\Omega+\omega_{m}\right)}+\left(c_{49}-\mathrm{i} c_{50}\right) A^{2} e^{2 \mathrm{i} \omega_{m} T_{0}} \\
& +\left(c_{51}+\mathrm{i} c_{52}\right) A^{2} e^{-2 \mathrm{i} T_{0}\left(\Omega-\omega_{m}\right)}+c_{53} A \bar{A} \\
& +4 \xi \alpha \omega_{m}^{2} \Omega^{2}\left(\Gamma_{1} c_{21}-\Gamma_{2} c_{22}\right) / \omega_{e}^{4}+\text { c.c. } \\
& q_{4}=\left(c_{60}+\mathrm{i} c_{61}\right) A e^{-\mathrm{i} T_{0}\left(3 \Omega-\omega_{m}\right)}+\left(c_{62}+\mathrm{i} c_{63}\right) A e^{3 \mathrm{i} T_{0}\left(3 \Omega+\omega_{m}\right)} \\
& +\left(c_{64}+\mathrm{i} c_{65}\right) A^{3} e^{\mathrm{i} T_{0}\left(\Omega+3 \omega_{m}\right)}+\left(c_{66}+\mathrm{i} c_{67}\right) A^{3} e^{-\mathrm{i} T_{0}\left(\Omega-3 \omega_{m}\right)} \\
& \left(c_{68}+\mathrm{i} c_{69}+\left(c_{70}+\mathrm{i} c_{71}\right) A \bar{A}\right) A e^{\mathrm{i} T_{0}\left(\Omega+\omega_{m}\right)} \\
& +\left(c_{72}+\mathrm{i} c_{73}+\left(c_{74}+\mathrm{i} c_{75}\right) A \bar{A}\right) A e^{-\mathrm{i} T_{0}\left(\Omega-\omega_{m}\right)}+\text { c.c. }
\end{aligned}
$$

Moving to the fifth order, the solvability condition for the mechanical amplitude, obtained by means of Eqs. (8), $(10), \sqrt{11}),(12),(13), \sqrt{14}),(26),(27)$, is expressed as follows:

$$
D_{4} A=\left(c_{80}+\mathrm{i} c_{81}\right) A+\left(c_{82}+\mathrm{i} c_{83}\right) A^{2} \bar{A}
$$

Derivatives of the mechanical amplitude with respect to time scales $T_{3}(26)$ and $T_{4}(28)$ are added to Eq. (15), and after reabsorption of $\epsilon$ parameter the complex mechanical amplitude becomes

$$
\begin{aligned}
& \dot{A}=\frac{1}{\omega_{e}^{4}} A\left(\xi \alpha \omega _ { m } ^ { 3 } \Omega \left(-\mathrm{i} \Omega\left(\Gamma_{1}\left(c_{14}+c_{16}+\mathrm{i}\left(c_{15}-c_{17}\right)\right)+\Gamma_{2}\left(c_{15}+c_{17}+\mathrm{i}\left(c_{16}-c_{14}\right)\right)\right)\right.\right. \\
& \left.+\omega_{m}\left(-\Gamma_{2}\left(c_{14}+c_{16}+\mathrm{i}\left(c_{15}-c_{17}\right)\right)+\Gamma_{1}\left(c_{15}+c_{17}+\mathrm{i}\left(c_{16}-c_{14}\right)\right)\right)\right) \\
& \left.+\left(c_{80}+\mathrm{i} c_{81}\right) \omega_{e}^{4}-\zeta_{m} \omega_{m} \omega_{e}^{4}\right)+\left(c_{82}+\mathrm{i} c_{83}\right) A^{2} \bar{A}
\end{aligned}
$$

which, in Cartesian coordinates reads

$$
\begin{aligned}
& \dot{r}=d_{1} r^{3}+d_{2} s^{3}+d_{1} r s^{2}+d_{2} r^{2} s+d_{3} r+d_{4} s \\
& \dot{s}=-d_{2} r^{3}+d_{1} s^{3}-d_{2} r s^{2}+d_{1} r^{2} s-d_{4} r+d_{3} s
\end{aligned}
$$

With respect to the AMEs obtained at the third order (17), system (30) is enriched by the cubic terms furnished by the contribution of the fifth order secular terms (28), which also modify the expressions of the linear coefficients $d_{3}, d_{4}$. As far as the reconstructed solutions up to the third order of Eq. (19), the fourth order solutions (27) show to alter the constant term of the mechanical amplitude, while no enrichment is provided to the terms of frequency $\Omega$ in the electrical solution. Consequently, the updated amplitude of the mechanical variable becomes

$$
X=c_{13}+\frac{1}{4} c_{53}\left(r^{2}+s^{2}\right)+\frac{4 \xi \alpha \Omega^{2} \omega_{m}^{2}}{\omega_{e}^{4}}\left(c_{21} \Gamma_{1}-c_{22} \Gamma_{2}\right)+\sqrt{r^{2}+s^{2}}
$$




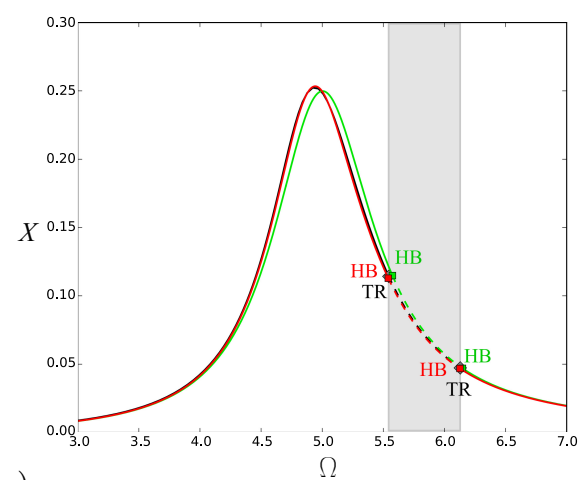

(a)

Figure 6: Comparison between numerical (black), third order asymptotic (green) and fifth order asymptotic (red) responses with respect to the mechanical amplitude $X$ (a) and the electrical amplitude $Q$ (b), with detection of instability regions, for $\mu=0.2$ and $\beta=1.0$. Gray diamond: torus bifurcation; green/red square: Hopf bifurcation.

which, together with the asymptotic system (30), provides the results represented with red curves in Fig. 6. The comparison with the numerical outcomes (black) and with that of the third-order AMEs (green), displays a further improvement in the accuracy of the analytical solutions mostly pertaining, as expected, to the mechanical response, which now reproduces almost perfectly the numerical curve. The enhancement in the detection of the bifurcations marking the unstable region can be also observed. The latter aspect can be better observed in Fig. 7 where the bifurcation threshold, with the related enlargement, is portrayed in the $\Omega-\alpha$ plane. The unstable region lower bound is more precisely detected by the fifth-order AMEs (dotted red) with respect to the third-order ones (dashed green), especially with regard to the right branch which is coinciding with the numerical results (solid gray). Moreover, differently from the third order case (Fig. 5), the left branch is better approximated, with a good precision for values of the coupling parameter $\alpha$ lower than 0.15 , and with an opposite trend with respect to the third-order threshold as $\alpha$ increases.

System (30) allows to describe also the behavior inside the unstable region, where numerical investigations point out the presence of a confined region of quasiperiodic solutions, as shown by the Poincaré maps at different values of the forcing frequency represented in gray in Fig. 8 . Here, the maximum and minimum values of the switched branch arising from the asymptotically detected Hopf bifurcations are reported too (red curves), highlighting a very good accordance between numerical and analytical results, from both a qualitative and quantitative viewpoint. In fact, AMEs 30 enable us to accurately catch the amplitudes of the quasiperiodic responses, and to capture their non-symmetric 180 evolution inside the detected region. The qualitative description of a quasiperiodic solution (Fig. 9p and of a 6-period solution (Fig. 10p occurring inside the investigated region is shown. The asymptotic system, beyond confirming the accuracy of the amplitude values obtained, also with respect to the time evolution of the solutions (red curves in figure), allow to detect the qualitative difference 


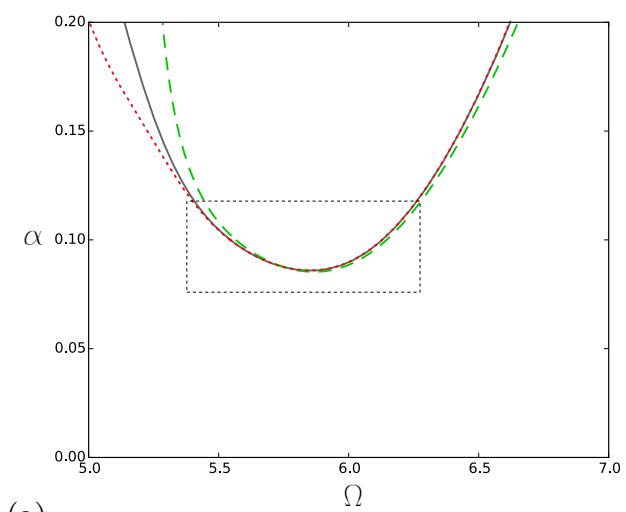

(a)

Figure 7: Third order asymptotic (dashed green), fifth order asymptotic (dotted red) and numerical (solid gray) stability thresholds in the $\Omega-\alpha(\beta)$ plane (a), and enlargement (b), at $\frac{\omega_{m}}{\omega_{e}}=\mu=0.2$.

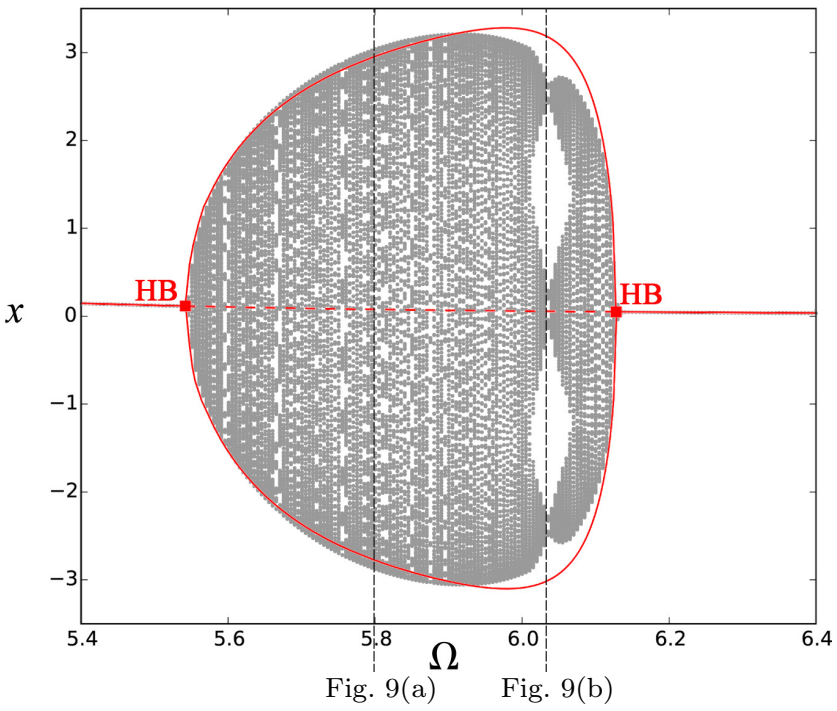

Figure 8: Comparison between fifth order asymptotic amplitude (red) and numerical Poincaré maps of the mechanical quasiperiodic solutions, at $\alpha=0.1$.

between mechanical and electrical responses. As a matter of fact, the electrical phase portraits, and the relevant FFT, are markedly quasiperiodic (Fig. 9) and highly periodic (Fig. 10), while the mechanical response is always dominated by a single frequency corresponding to its natural frequency. This also explains the lack of oscillating responses in the post-critical behavior provided by the asymptotic system (see red curves of Figs. 8, 90 which, as already observed, provides information about the sole mechanical amplitude. In this regard, it is worth noting that, coherently with the obtained results, while the analytical characterization of the critical points 20 would suggest the presence of a 

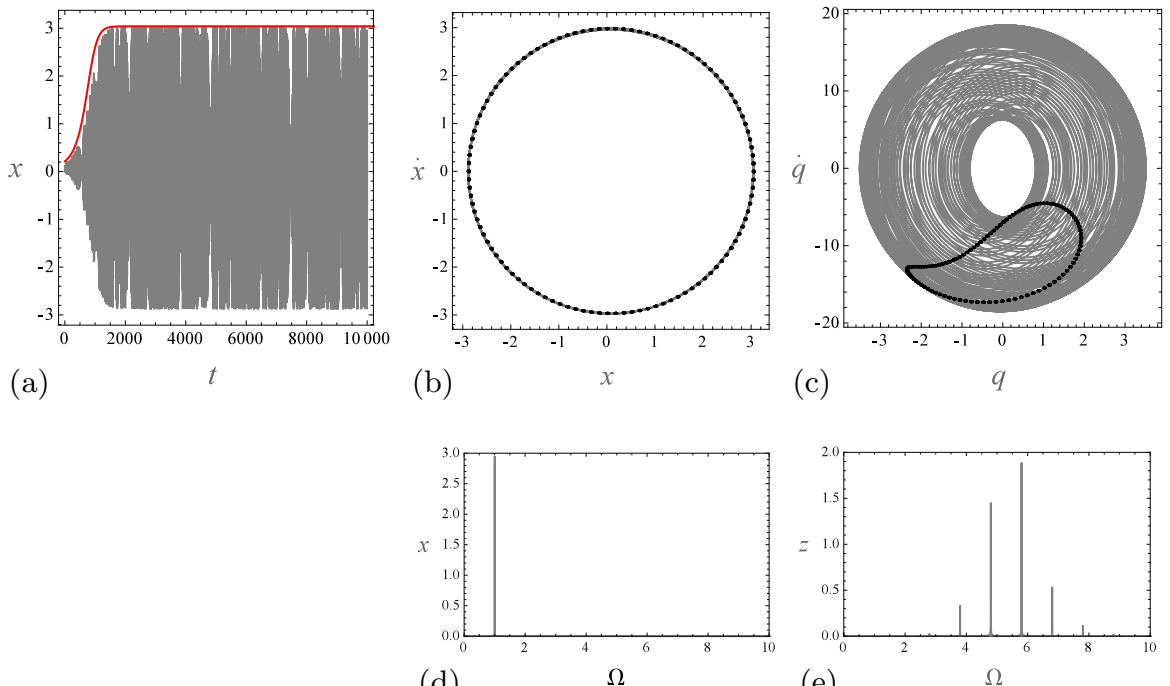

(e)

Figure 9: Characterization of the quasiperiodic response at $\Omega=5.8$ and $\alpha=0.1$. Temporal evolution (a), phase portraits with Poincaré maps in the mechanical (b) and electrical (c) planes, and FFT of the mechanical (d) and electrical (e) response. In (a), the comparison between the numerical (gray) responses and the fifth order asymptotic (red) amplitudes is reported.

pitchfork bifurcation, Hopf bifurcations are here detected. The reason of this iscrepancy is related to the Cartesian coordinates choice. These coordinates are convenient to study systems with trivial solution, however, differently form the pol a ones, they do not filter out the oscillating component of the response. Nevertheless, the stability analysis herein developed is consistent with the actual system behavior which, due to the contribution of the electrical solution, confirms the occurrence of a torus bifurcation, corresponding to a Hopf in the relevant asymptotic model.

\section{Conclusions}

The weakly nonlinear dynamics of a linear oscillator nonlinearly coupled with a linear electric circuit is studied in conditions of primary electrical resonance. Willing to investigate the stability of the frequency-amplitude responses,the multiple scale analysis up to the third order is applied to the system of 2 d.o.f. ODEs to obtain Cartesian Amplitude Modulation Equations (AMEs) enabling to reconstruct the particular mechanical and electrical solutions at each scaling order.

210 The validation, carried out by comparison with the numerical integration, confirms the accuracy of the asymptotic approach in qualitatively and quantitatively grasping the mechanical and electrical responses of the coupled system, and their stability regions. Moreover, the analytical expression of the critical 

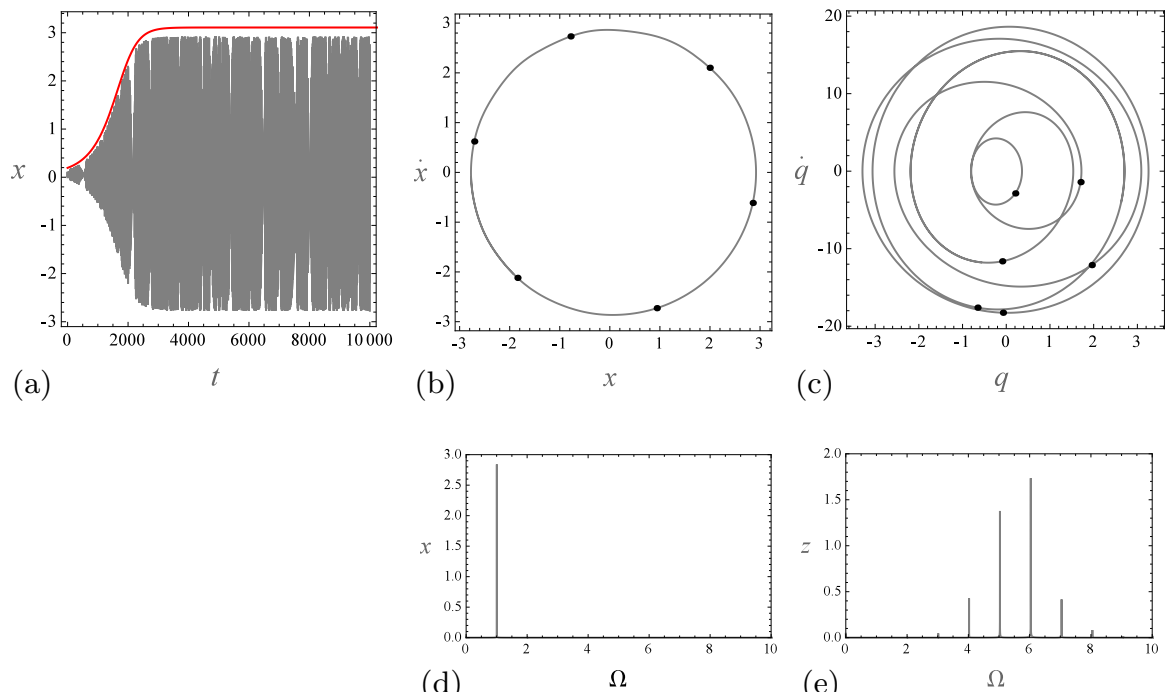

(e)

Figure 10: Characterization of the 6-period response at $\Omega=6.036$ and $\alpha=0.1$. Temporal evolution (a), phase portraits with Poincaré maps in the mechanical (b) and electrical (c) planes, and FFT of the mechanical (d) and electrical (e) response. In (a), the comparison between the numerical (gray) responses and the fifth order asymptotic (red) amplitudes is reported.

stability threshold allows to verify the influence of the main parameters on the system dynamical response; stability charts are also derived to properly tune the system parameters in order to predict and control the onset of instability.

With the aim to achieve a comprehensive description of the system dynamics, the post-critical behavior is also investigated by enriching the asymptotic approach with two further orders. The ensuing fifth order AMEs allow to catch rather accurately the numerical amplitudes of the quasiperiodic solutions originating from the torus bifurcations of the ODEs system, as well as to improve further the third order results related to the description of the frequency-response curves.

\section{Acknowledgements}

VS acknowledges the financial support of PRIN 2015 N. 2015JW9NJT. FR acknowledges the financial support of PRIN 2015 N. 2015TTJN95. 


\section{Appendix A.}

Expressions of $c_{i j}$ and $f_{i}(\Omega)$ coefficients introduced in Sect. 3

$$
\begin{aligned}
& c_{11}=\frac{\xi \alpha\left(\Gamma_{1}-\Gamma_{2}\right)\left(\Gamma_{1}+\Gamma_{2}\right) \Omega^{2} \omega_{m}^{4}}{\left(4 \Omega^{2}-\omega_{m}^{2}\right) \omega_{e}^{4}} \\
& c_{12}=\frac{2 \xi \alpha \Gamma_{1} \Gamma_{2} \Omega^{2} \omega_{m}^{4}}{\left(4 \Omega^{2}-\omega_{m}^{2}\right) \omega_{e}^{4}} \\
& c_{13}=\frac{2 \xi \alpha\left(\Gamma_{1}^{2}+\Gamma_{2}^{2}\right) \Omega^{2} \omega_{m}^{2}}{\omega_{e}^{4}} \\
& c_{14}=\frac{\alpha \Omega\left(\Omega+\omega_{m}\right)\left(-\Gamma_{1}\left(\Omega+\omega_{m}\right)^{2}+2 \Gamma_{2} \zeta_{e} \omega_{e}\left(\Omega+\omega_{m}\right)+\omega_{e}^{2} \Gamma_{1}\right)}{\left(\Omega+\omega_{m}\right)^{4}+2\left(2 \zeta_{e}^{2}-1\right)\left(\Omega+\omega_{m}\right)^{2} \omega_{e}^{2}+\omega_{e}^{4}} \\
& c_{15}=\frac{\alpha \Omega\left(\Omega+\omega_{m}\right)\left(-\Gamma_{2}\left(\Omega+\omega_{m}\right)^{2}-2 \Gamma_{1} \zeta_{e} \omega_{e}\left(\Omega+\omega_{m}\right)+\omega_{e}^{2} \Gamma_{2}\right)}{\left(\Omega+\omega_{m}\right)^{4}+2\left(2 \zeta_{e}^{2}-1\right)\left(\Omega+\omega_{m}\right)^{2} \omega_{e}^{2}+\omega_{e}^{4}} \\
& c_{16}=\frac{\alpha \Omega\left(\Omega-\omega_{m}\right)\left(-\Gamma_{1}\left(\Omega-\omega_{m}\right)^{2}+2 \Gamma_{2} \zeta_{e} \omega_{e}\left(\Omega-\omega_{m}\right)+\omega_{e}^{2} \Gamma_{1}\right)}{\left(\Omega-\omega_{m}\right)^{4}+2\left(2 \zeta_{e}^{2}-1\right)\left(\Omega-\omega_{m}\right)^{2} \omega_{e}^{2}+\omega_{e}^{4}} \\
& c_{17}=\frac{\alpha \Omega\left(\Omega-\omega_{m}\right)\left(-\Gamma_{2}\left(\Omega-\omega_{m}\right)^{2}-2 \Gamma_{1} \zeta_{e} \omega_{e}\left(\Omega-\omega_{m}\right)+\omega_{e}^{2} \Gamma_{2}\right)}{\left(\Omega-\omega_{m}\right)^{4}+2\left(2 \zeta_{e}^{2}-1\right)\left(\Omega-\omega_{m}\right)^{2} \omega_{e}^{2}+\omega_{e}^{4}} \\
& c_{21}=\left(\alpha \Omega ^ { 2 } \left(\left(\Gamma_{1}\left(c_{11}-c_{13}\right)+c_{12} \Gamma_{2}\right) \Omega^{2}+2\left(\left(c_{11}+c_{13}\right) \Gamma_{2}-c_{12} \Gamma_{1}\right) \zeta_{e} \Omega \omega_{e}\right.\right. \\
& \left.\left.-\left(\left(c_{11}-c_{13}\right) \Gamma_{1}+c_{12} \Gamma_{2}\right) \omega_{e}^{2}\right)\right) /\left(\Omega^{4}+2\left(2 \zeta_{e}^{2}-1\right) \Omega^{2} \omega_{e}^{2}+\omega_{e}^{4}\right) \\
& c_{22}=-\left(\alpha \Omega ^ { 2 } \left(\left(-\Gamma_{2}\left(c_{11}+c_{13}\right)+c_{12} \Gamma_{1}\right) \Omega^{2}+2\left(\left(c_{11}-c_{13}\right) \Gamma_{1}+c_{12} \Gamma_{2}\right) \zeta_{e} \Omega \omega_{e}\right.\right. \\
& \left.\left.-\left(\left(c_{11}-c_{13}\right) \Gamma_{1}+c_{12} \Gamma_{2}\right) \omega_{e}^{2}\right)\right) /\left(\Omega^{4}+2\left(2 \zeta_{e}^{2}-1\right) \Omega^{2} \omega_{e}^{2}+\omega_{e}^{4}\right) \\
& c_{22}=-\left(\alpha \Omega ^ { 2 } \left(\left(-\Gamma_{2}\left(c_{11}+c_{13}\right)+c_{12} \Gamma_{1}\right) \Omega^{2}+2\left(\left(c_{11}-c_{13}\right) \Gamma_{1}+c_{12} \Gamma_{2}\right) \zeta_{e} \Omega \omega_{e}\right.\right. \\
& \left.\left.+\left(\left(c_{11}+c_{13}\right) \Gamma_{2}-c_{12} \Gamma_{1}\right) \omega_{e}^{2}\right)\right) /\left(\Omega^{4}+2\left(2 \zeta_{e}^{2}-1\right) \Omega^{2} \omega_{e}^{2}+\omega_{e}^{4}\right) \\
& c_{23}=\frac{3 \alpha \Omega^{2}\left(9\left(-c_{11} \Gamma_{1}+c_{12} \Gamma_{2}\right) \Omega^{2}+6\left(c_{12} \Gamma_{1}+c_{11} \Gamma_{2}\right) \zeta_{e} \omega \omega_{e}+\left(c_{11} \Gamma_{1}-c_{12} \Gamma_{2}\right) \omega_{e}^{2}\right.}{81 \Omega^{4}+18\left(2 \zeta_{e}^{2}-1\right) \Omega^{2} \omega_{e}^{2}+\omega_{e}^{4}} \\
& c_{24}=-\frac{3 \alpha \Omega^{2}\left(9\left(c_{12} \Gamma_{1}+c_{11} \Gamma_{2}\right) \Omega^{2}+6\left(c_{11} \Gamma_{1}-c_{12} \Gamma_{2}\right) \zeta_{e} \omega \omega_{e}-\left(c_{12} \Gamma_{1}+c_{11} \Gamma_{2}\right) \omega_{e}^{2}\right.}{81 \Omega^{4}+18\left(2 \zeta_{e}^{2}-1\right) \Omega^{2} \omega_{e}^{2}+\omega_{e}^{4}} \\
& c_{25}=-\frac{\alpha\left(\Omega-2 \omega_{m}\right)\left(\Omega-\omega_{m}\right)\left(c_{16}\left(\Omega-2 \omega_{m}\right)^{2}-2 c_{17} \zeta_{e}\left(\Omega-2 \omega_{m}\right) \omega_{e}-c_{16} \omega_{e}^{2}\right)}{\left(\Omega-2 \omega_{m}\right)^{4}+2\left(\zeta_{e}^{2}-1\right)\left(\Omega-2 \omega_{m}\right)^{2} \omega_{e}^{2}+\omega_{e}^{4}} \\
& c_{26}=-\frac{\alpha\left(\Omega-2 \omega_{m}\right)\left(\Omega-\omega_{m}\right)\left(c_{17}\left(\Omega-2 \omega_{m}\right)^{2}-2 c_{16} \zeta_{e}\left(\Omega-2 \omega_{m}\right) \omega_{e}-c_{17} \omega_{e}^{2}\right)}{\left(\Omega-2 \omega_{m}\right)^{4}+2\left(\zeta_{e}^{2}-1\right)\left(\Omega-2 \omega_{m}\right)^{2} \omega_{e}^{2}+\omega_{e}^{4}}
\end{aligned}
$$




$$
\begin{aligned}
& c_{27}=\frac{\alpha\left(\Omega+2 \omega_{m}\right)\left(\Omega+\omega_{m}\right)\left(-c_{14}\left(\Omega+2 \omega_{m}\right)^{2}+2 c_{15} \zeta_{e}\left(\Omega+2 \omega_{m}\right) \omega_{e}+c_{14} \omega_{e}^{2}\right)}{\left(\Omega+2 \omega_{m}\right)^{4}+2\left(\zeta_{e}^{2}-1\right)\left(\Omega+2 \omega_{m}\right)^{2} \omega_{e}^{2}+\omega_{e}^{4}} \\
& c_{28}=\frac{\alpha\left(\Omega+2 \omega_{m}\right)\left(\Omega+\omega_{m}\right)\left(c_{15}\left(\Omega+2 \omega_{m}\right)^{2}+2 c_{14} \zeta_{e}\left(\Omega+2 \omega_{m}\right) \omega_{e}-c_{15} \omega_{e}^{2}\right)}{\left(\Omega+2 \omega_{m}\right)^{4}+2\left(\zeta_{e}^{2}-1\right)\left(\Omega+2 \omega_{m}\right)^{2} \omega_{e}^{2}+\omega_{e}^{4}} \\
& c_{29}=\frac{\alpha \Omega}{\Omega^{4}+2\left(\zeta_{e}^{2}-1\right) \Omega^{2} \omega_{e}^{2}+\omega_{e}^{4}}\left(-\Omega^{2}\left(\left(c_{14}+c_{16}\right) \Omega+\left(c_{14}-c_{16}\right) \omega_{m}\right)\right. \\
& \left.+2 \zeta_{e} \Omega\left(\left(c_{15}+c_{17}\right) \Omega+\left(c_{15}-c_{17}\right) \omega_{m}\right) \omega_{e}+\left(\left(c_{14}+c_{16}\right) \Omega+\left(c_{14}-c_{16}\right) \omega_{m}\right) \omega_{e}^{2}\right) \\
& c_{30}=\frac{\alpha \Omega}{\Omega^{4}+2\left(\zeta_{e}^{2}-1\right) \Omega^{2} \omega_{e}^{2}+\omega_{e}^{4}}\left(\Omega^{2}\left(\left(c_{15}+c_{17}\right) \Omega+\left(c_{15}-c_{17}\right) \omega_{m}\right)\right. \\
& \left.+2 \zeta_{e} \Omega\left(\left(c_{14}+c_{16}\right) \Omega+\left(c_{14}-c_{16}\right) \omega_{m}\right) \omega_{e}-\left(\left(c_{15}+c_{17}\right) \Omega+\left(c_{15}-c_{17}\right) \omega_{m}\right) \omega_{e}^{2}\right) \\
& c_{31}=\frac{\xi \alpha\left(c_{14} \Gamma_{1}-c_{15} \Gamma_{2}\right) \omega_{m}^{4}}{2 \omega_{e}^{4}} \\
& c_{32}=\frac{\xi \alpha\left(c_{15} \Gamma_{1}+c_{14} \Gamma_{2}\right) \omega_{m}^{4}}{2 \omega_{e}^{4}} \\
& c_{33}=\frac{\xi \alpha\left(c_{16} \Gamma_{1}-c_{17} \Gamma_{2}\right) \omega_{m}^{4}}{2 \omega_{e}^{4}} \\
& c_{34}=\frac{\xi \alpha\left(c_{17} \Gamma_{1}+c_{16} \Gamma_{2}\right) \omega_{m}^{4}}{2 \omega_{e}^{4}} \\
& f_{1}(\Omega)=\left(\omega _ { m } ^ { 3 } \left(-\Omega^{2}\left(\Omega^{2}-\omega_{m}^{2}\right)^{4}-\left(-3+4 \zeta_{e}^{2}\right)\left(\Omega^{2}+\omega_{m}^{2}\right)\left(\Omega^{3}-\Omega \omega_{m}^{2}\right)^{2} \omega_{e}^{2}\right.\right. \\
& \left.\left.+\Omega^{2}\left(\left(-3+4 \zeta_{e}^{2}\right) \Omega^{4}-2\left(1+4 \zeta_{e}^{2}\right) \Omega^{2} \omega_{m}^{2}+\left(-3+4 \zeta_{e}^{2}\right) \omega_{m}^{4}\right) \omega_{e}^{4}+\Omega^{2}\left(\Omega^{2}+\omega_{m}^{2}\right) \omega_{e}^{6}\right)\right) / \\
& \left(2\left(\Omega^{4}+2\left(-1+2 \zeta_{e}^{2}\right) \Omega^{2} \omega_{e}^{2}+\omega_{e}^{4}\right)\left(\left(\Omega-\omega_{m}\right)^{4}+2\left(-1+2 \zeta_{e}^{2}\right)\left(\Omega-\omega_{m}\right)^{2} \omega_{e}^{2}+\omega_{e}^{4}\right)\right. \\
& \left.\left(\left(\Omega-\omega_{m}\right)^{4}+2\left(-1+2 \zeta_{e}^{2}\right)\left(\omega+\omega_{m}\right)^{2} \omega_{e}^{2}+\omega_{e}^{4}\right)\right) \\
& f_{2}(\Omega)=\left(\zeta_{e} \Omega^{2} \omega_{m}^{4} \omega_{e}\left(\left(\Omega^{2}-\omega_{m}^{2}\right)^{3}-2\left(-1+2 \zeta_{e}^{2}\right)\left(\Omega^{2}-\omega_{m}^{2}\right)^{2} \omega_{e}^{2}-\left(3 \Omega^{2}+\omega_{m}^{2}\right) \omega_{e}^{4}\right)\right) / \\
& \left(\left(\Omega^{4}+2\left(-1+2 \zeta_{e}^{2}\right) \Omega^{2} \omega_{e}^{2}+\omega_{e}^{4}\right)\left(\left(\Omega-\omega_{m}\right)^{4}+2\left(-1+2 \zeta_{e}^{2}\right)\left(\Omega-\omega_{m}\right)^{2} \omega_{e}^{2}+\omega_{e}^{4}\right)\right. \\
& \left.\left(\left(\Omega+\omega_{m}\right)^{4}+2\left(-1+2 \zeta_{e}^{2}\right)\left(\Omega+\omega_{m}\right)^{2} \omega_{e}^{2}+\omega_{e}^{4}\right)\right)
\end{aligned}
$$

\section{Appendix B.}

Expressions of $f_{i}$ and $g_{i}(i=4,5)$ coefficients introduced in Eqs. 23, 24,

$$
\begin{aligned}
& f_{4}\left(x_{1}, x_{2}, x_{3}, q_{1}, q_{2}, q_{3}\right)=-D_{1}^{2} x_{2}-2\left(D_{0} D_{1} x_{3}+2 D_{0} D_{2} x_{2}+D_{1} D_{2} x_{1}+D_{0} D_{3} x_{1}\right) \\
& -2 \zeta_{m} \omega_{m}\left(D_{0} x_{2}+D_{1} x_{1}\right)+\left(\xi \alpha \omega_{m}^{4}\right) / \omega_{e}^{4}\left(D_{0} q_{2}^{2}+D_{1} q_{1}^{2}\right. \\
& \left.+2 D_{0} q_{1} D_{0} q_{3}+2 D_{0} q_{2} D_{1} q_{1}+2 D_{0} q_{1} D_{1} q_{2}+2 D_{0} q_{1} D_{2} q_{1}\right)
\end{aligned}
$$




$$
\begin{aligned}
& g_{4}\left(x_{1}, x_{2}, x_{3}, q_{1}, q_{2}, q_{3}\right)=-D_{1}^{2} q_{2}-2 D_{0} D_{1} q_{3}-2 D_{0} D_{2} q_{2}-2 D_{1} D_{2} q_{1}-2 D_{0} D_{3} q_{1} \\
& -\alpha\left(D_{0}^{2} q_{3} x_{1}+D_{1}^{2} q_{1} x_{1}+D_{0}^{2} q_{2} x_{2}+D_{0}^{2} q_{1} x_{3}+D_{0} q_{3} D_{0} x_{1}+D_{0} q_{2} D_{0} x_{2}\right. \\
& +D_{0} q_{1} D_{0} x_{3}+D_{0} x_{2} D_{1} q_{1}+D_{0} x_{1} D_{1} q_{2}+D_{0} q_{2} D_{1} x_{1}+D_{1} q_{1} D_{1} x_{1}+D_{0} q_{1} D_{1} x_{2} \\
& \left.+D_{0} x_{1} D_{2} q_{1}+D_{0} q_{1} D_{2} x_{1}+2 D_{0} D_{1} q_{2} x_{1}+2 D_{0} D_{2} q_{1} x_{1}+2 D_{0} D_{1} q_{1} x_{2}\right) \\
& -2 \zeta_{e} \omega_{e}\left(D_{1} q_{3}+D_{2} q_{2}+D_{3} q_{1}\right) \\
& f_{5}\left(x_{1}, x_{2}, x_{3}, x_{4}, q_{1}, q_{2}, q_{3}, q_{4}\right)=-D_{1}^{2} x_{3}-D_{2}^{2} x_{1}-2\left(D_{0} D_{1} x_{4}+D_{0} D_{2} x_{3}\right. \\
& \left.+D_{1} D_{2} x_{2}+D_{0} D_{3} x_{2}+D_{1} D_{3} x_{1}+D_{0} D_{4} x_{1}\right)-2 \zeta_{m} \omega_{m}\left(D_{0} x_{3}+D_{1} x_{2}+D_{2} x_{1}\right) \\
& +\left(2 \xi \alpha \omega_{m}^{4}\right) / \omega_{e}^{4}\left(D_{0} q_{2} D_{0} q_{3}+D_{0} q_{1} D_{0} q_{4}+D_{0} q_{3} D_{1} q_{1}+D_{0} q_{2} D_{1} q_{2}+D_{1} q_{1} D_{1} q_{2}\right. \\
& \left.+D_{0} q_{1} D_{1} q_{3}+D_{0} q_{2} D_{2} q_{1}+D_{1} q_{1} D_{2} q_{1}+D_{0} q_{1} D_{2} q_{2}+D_{0} q_{1} D_{3} q_{1}\right) \\
& g_{5}\left(x_{1}, x_{2}, x_{3}, x_{4}, q_{1}, q_{2}, q_{3}, q_{4}\right)=-D_{1}^{2} q_{3}-D_{2}^{2} q_{1}-2\left(D_{0} D_{1} q_{4}+D_{0} D_{2} q_{3}\right. \\
& \left.+D_{1} D_{2} q_{2}+D_{0} D_{3} q_{2}+D_{1} D_{3} q_{1}+D_{0} D_{4} q_{1}\right)-\alpha\left(D_{1}^{2} q_{2} x_{1}+D_{1}^{2} q_{1} x_{2}\right. \\
& +D_{0}^{2} q_{2} x_{3}+D_{0}^{2} q_{1} x_{4}+D_{0}^{2} q_{3} x_{2}+D_{0}^{2} q_{4} x_{1}+D_{0} q_{4} D_{0} x_{1}+D_{0} q_{3} D_{0} x_{2} \\
& +D_{0} q_{2} D_{0} x_{3}+D_{0} q_{1} D_{0} x_{4}+D_{0} x_{3} D_{1} q_{1}+D_{0} x_{2} D_{1} q_{2}+D_{0} x_{1} D_{1} q_{3} \\
& +D_{0} q_{3} D_{1} x_{1}+D_{1} q_{2} D_{1} x_{1}+D_{0} q_{2} D_{1} x_{2}+D_{1} q_{1} D_{1} x_{2}+D_{0} q_{1} D_{1} x_{3} \\
& +D_{0} x_{2} D_{2} q_{1}+D_{1} x_{1} D_{2} q_{1}+D_{0} x_{1} D_{2} q_{2}+D_{0} q_{2} D_{2} x_{1}+D_{1} q_{1} D_{2} x_{1}+D_{0} q_{1} D_{2} x_{2} \\
& +D_{0} x_{1} D_{3} q_{1}+D_{0} q_{1} D_{3} x_{1}+2 D_{0} D_{1} q_{3} x_{1}+2 D_{0} D_{2} q_{2} x_{1}+2 D_{1} D_{2} q_{1} x_{1} \\
& \left.+2 D_{0} D_{3} q_{1} x_{1}+2 D_{0} D_{1} q_{2} x_{2}+2 D_{0} D_{2} q_{1} x_{2}+2 D_{0} D_{1} q_{1} x_{3}\right) \\
& -2 \zeta_{e} \omega_{e}\left(D_{1} q_{4}+D_{2} q_{3}+D_{3} q_{2}+D_{4} q_{1}\right)
\end{aligned}
$$

\section{References}

[1] R. H. Bishop, Mechatronic systems, sensors, and actuators: fundamentals and modeling, CRC press, 2007.

[2] M. F. Rahman, N. C. Cheung, K. W. Lim, Position estimation in solenoid actuators, IEEE Trans Ind Appl 32 (3) (1996) 552-559.

[3] V. Giurgiutiu, S. E. Lyshevski, Micromechatronics: Modeling, analysis, and design with MATLAB, CRC Press, 2016.

[4] R. K. Govindarajan, G. Venkataramanan, Servo control of solenoid actuators using augmented feedback linearization, in: Control and Modeling for Power Electronics (COMPEL), 2017 IEEE 18th Workshop on, IEEE, 2017, pp. 1-6. 
[5] A. Dobrucki, B. Merit, V. Lemarquand, G. Lemarquand, Modeling of the nonlinear distortion in electrodynamic loudspeakers caused by the voicecoil inductance, in: 10ème Congrès Français d'Acoustique, 2010.

[6] N. Kesavamurthy, G. Rao, A study of bethenod's phenomenon, IEEE Trans Circuit Theory 19 (2) (1972) 215-218.

[7] D. D. Kana, Parametric coupling in a nonlinear electromechanical system, J Eng Ind 89 (4) (1967) 839-847.

[8] P. S. Landa, Y. B. Duboshinskiǔ, Self-oscillatory systems with highfrequency energy sources, Sov Phys Uspekhi 32 (8) (1989) 723.

[9] P. S. Landa, Nonlinear oscillations and waves in dynamical systems, Vol. 360, Springer Science \& Business Media, 2013.

[10] P. Koval'chuk, L. Kruk, Forced nonlinear vibrations of cylindrical shells filled with a liquid, Int Appl Mech 38 (11) (2002) 1388-1393.

[11] I. T. Georgiou, F. Romeo, Multi-physics dynamics of a mechanical oscillator coupled to an electro-magnetic circuit, Int J Nonlin Mech 70 (2015) 153164 .

[12] F. Romeo, I. T. Georgiou, Multiphysics chaotic interaction in a coupled electro-magneto-mechanical system, in: ASME Proceedings IMECE, 2014, p. V04BT04A018.

[13] A. Luongo, D. Zulli, G. Piccardo, Analytical and numerical approaches to nonlinear galloping of internally resonant suspended cables, J Sound Vib 315 (3) (2008) 375-393.

[14] V. Settimi, O. Gottlieb, G. Rega, Asymptotic analysis of a noncontact AFM microcantilever sensor with external feedback control, Nonlinear Dynam 79 (4) (2015) 2675-2698.

[15] J. Warminski, D. Zulli, G. Rega, J. Latalski, Revisited modelling and multimodal nonlinear oscillations of a sagged cable under support motion, Meccanica 51 (11) (2016) 2541-2575.

[16] A. H. Nayfeh, Perturbation methods, John Wiley \& Sons, 2008. 